\title{
Noncommutative Field Theories and Integrable Models in 2d
}

\author{
Marco Moriconi* \\ Newman Laboratory of Nuclear Studies, Cornell University \\ Ithaca, New York 14853, USA \\ and \\ Instituto de Física \\ Universidade Federal do Rio de Janeiro \\ Rio de Janeiro, RJ 21945-970, Brazil \\ E-mail: marco@if.ufrj.bri

\section{Cabrera-Carnero} \\ IFT, Unesp \\ Rua Pamplona, 145 \\ São Paulo, SP 01405-900, Brazil \\ E-mail: 'cabrera@ift.unesp.bri
}

\begin{abstract}
We study the noncommutative extensions of certain integrable field theories, namely the sine- and sinh-Gordon (sG and shG) models, and the $U(N)$ principal chiral model $(\mathrm{pcm})$. We argue that the Moyal deformations of the $\mathrm{sG}$ and shG models are not integrable, by looking at tree-level amplitudes where there is particle production. By considering the noncommutative generalization of the zero-curvature method, it is possible to define integrable versions of the noncommutative $\mathrm{sG}$ and shG models, which introduce extra constraints. The noncommutative pcm is shown to be integrable and we discuss the existence of non-trivial non-local conserved charges, and the associated noncommutative zero-curvature condition.
\end{abstract}

\section{Introduction}

In this note we are going to summarize the results described in iin. The presentation will be rather informal and we refer the interested reader to that reference in order to see a more complete discussion.

Noncommutative field theories (ncfts) have attracted a great deal of attention in the past few years due to several reasons. First and foremost, its connection to string theory,

${ }^{*}$ Speaker. 
where they arise as certain limits of type IIB theories with a B field turned on [2] $]$, they serve as toy-models for the study of quantum gravity since they are intrinsically nonlocal, and a connection between noncommutative Chern-Simons theory and the fractional quantum Hall effect has been suggested by Susskind [i] $]$. Finally, there is a host of theoretical phenomena which are rather different from usual quantum field theory (qft), making them very appealing from the theoretical point of view

We will be considering euclidean field theories, since it has been pointed out that the introduction of space and time noncommutativity may lead to violations of unitarity It is conceivable tough, that some specific models could evade these arguments [i, i i i].

\section{Generalities on Noncommutative Field Theories}

We can define a noncommutative deformation of a given model, defined by some local lagrangian, by replacing the products of fields by the Moyal product, also known as the $\star$-product,

$$
\phi(x) \eta(x) \rightarrow \phi(x) \star \eta(x)=\left.\exp \left(\frac{i}{2} \theta^{\mu \nu} \partial_{\mu}^{z_{1}} \partial_{\nu}^{z_{2}}\right) \phi\left(z_{1}\right) \eta\left(z_{2}\right)\right|_{z_{1}=z_{2}=x}
$$

Once this has been done, we can deform the whole action and from there, derive the new Feynman rules $[\overline{8}, \bar{g}, \bar{g}]$. The first important point to notice is that quadratic terms in the action are equivalent to non-deformed terms, provided the fields fall fast enough at infinity and that we work in manifolds without boundaries and with trivial topology. This is due to the fact that $\phi(x) \star \eta(x)=\phi(x) \eta(x)+\partial_{\mu} T^{\mu}(x)$ for some (nonlocal) operator $T^{\mu}(x)$. Therefore the propagator of a ncft is the same as the non-deformed version $(\theta=0)$. The only difference will be in the vertices of the theory. One point that should be made though, is that in most cases the measure of the path integral is taken to be the same for $\theta \neq 0$ as the one for $\theta=0$, which amounts to saying that the vacuum of the deformed theory is the same as the one of the non-deformed theory. This can be established perturbatively, but one should be aware of possible complications due to non-perturbative effects.

We are going to use $\mathrm{a} \star$ subscript to indicate that we are considering Moyal products. For example $\phi_{\star}^{4}=\phi \star \phi \star \phi \star \phi$.

Let us analyze the change in the case of the $\phi^{4}$ and $\phi^{6}$ vertices. In momentum space the space-time integral of the Moyal product of a string of fields becomes

$$
\int d x \phi_{\star}^{n}=\int d p_{1} d p_{2} \ldots d p_{n} \exp \left(-\frac{i}{2} \sum_{i<j}\left(p_{i}\right)_{\mu} \theta^{\mu \nu}\left(p_{j}\right)_{\nu}\right) \phi\left(p_{1}\right) \phi\left(p_{2}\right) \ldots \phi\left(p_{n}\right) \delta\left(p_{1}+p_{2}+\ldots+p_{n}\right)
$$

We will introduce now the following convention: $\left.\frac{1}{2}\left(p_{i}\right)_{\mu} \theta^{\mu \nu}\left(p_{j}\right)_{\nu} \equiv p_{i} \wedge p_{j}\right)^{1}$. In the case of scalar $\phi^{4}$ vertex, this expression can be simplified by symmetrizing it over the momenta

$$
\begin{aligned}
\int d x \phi_{\star}^{4}= & \frac{1}{4 !} \sum_{\text {perm. }} \exp \left(-i \sum_{i<j} p_{i} \wedge p_{j}\right)=\frac{1}{3}\left(\cos \left(p_{1} \wedge p_{2}\right) \cos \left(p_{3} \wedge p_{4}\right)+\right. \\
& \left.\cos \left(p_{1} \wedge p_{3}\right) \cos \left(p_{2} \wedge p_{4}\right)+\cos \left(p_{1} \wedge p_{4}\right) \cos \left(p_{2} \wedge p_{3}\right)\right) .
\end{aligned}
$$

\footnotetext{
${ }^{1}$ Note a difference of a factor of $\frac{1}{2}$ from the usual convention in the literature.
} 
The $\phi^{6}$ vertex becomes

$$
\int d x \phi_{\star}^{6}=\frac{1}{6 !} \sum_{p e r m .} \exp \left(-i \sum_{i<j} p_{i} \wedge p_{j}\right)
$$

Therefore all one needs to do in order to compute anything perturbatively is to replace the vertices of a given Feynman diagram by their noncommutative expressions, like 2.31 and 2..4, and use the same propagators as in the commutative theory.

\section{Integrable Field Theory}

An integrable field theory is characterized by an infinite number of conserved charges in involution. This imposes severe constraints in the theory: there is no particle production in scattering processes, the set of in- and out-momenta is the same, the amplitude for $n$ particle scattering processes can be factorized in terms of two-body processes, introducing the all important two-body $S$-matrix $S_{2}$. The two-body $S$-matrix satisfies the Yang-Baxter equation (YBe) as a consequence of integrability. The YBe is trivial for theories where $S_{2}$ is a collection of phases, but it is highly non-trivial for theories where $S_{2}$ has a non-diagonal structure. This makes integrable field theories more controlled technically, without making them trivial [1] 10 , in a great number of different problems, in statistical mechanics, condensed matter, and high-energy physics, via string theory.

Out of the consequences of integrable field theories, we can say that the hallmark of integrability is the non-production of particles in any scattering process. This has to be true to all orders in perturbation theory, which in a loop expansion means that the tree-level amplitudes for particle production must vanish. This encodes the fact that the classical theory is integrable.

The models we are going to study are: sine-Gordon (sG), sinh-Gordon (shG), and principal chiral model (pcm). The $\mathrm{sG}$ model is defined by the following lagrangian

$$
\mathcal{L}_{s G}=\frac{1}{2}(\partial \phi)^{2}+\frac{m^{2}}{\beta^{2}}(\cos (\beta \phi)-1) .
$$

The shG model is obtained from the $\mathrm{sG}$ model by replacing $\beta \rightarrow i \beta$,

$$
\mathcal{L}_{s h G}=\frac{1}{2}(\partial \phi)^{2}+\frac{m^{2}}{\beta^{2}}(1-\cosh (\beta \phi)) .
$$

Finally, the pcm is defined through the following lagrangian

$$
\mathcal{L}_{p c m}=\frac{1}{2 g_{0}^{2}} \operatorname{Tr}\left(\partial^{\mu} \mathrm{g}^{-1} \partial_{\mu} \mathrm{g}\right)
$$

where $g$ is a group element, of one of the classical groups. Here we are going to consider only the $U(N)$ group, due to reasons that will become clear later. We only stress the fact that noncommutativity imposes constraints that restrict the existence of certain groups [1] 1 ind $]$. 
The sG and shG models are related by a simple replacement in the coupling constant, but have very different vacuum structure. In the shG model there is a single minimum, whereas in the sG model there is an infinite degeneracy, implying the existence of solitons. In any case, since the scattering amplitudes are analytical in the coupling constant, we can restrict our attention to the shG model, for simplicity (see [i] 3 in for a discussion of the noncommutative sine-Gordon model).

We will consider a simple scattering process of 2 particles going into 4 in the shG model at tree-level, whose amplitude is denoted by $\mathcal{M}_{2 \rightarrow 4}$. For this computation we can truncate the lagrangian

$$
\mathcal{L}_{\text {shG }}^{(6)}=\frac{1}{2}(\partial \phi)^{2}-\frac{m^{2}}{2} \phi^{2}-\frac{m^{2} \beta^{2}}{4 !} \phi^{4}-\frac{m^{2} \beta^{4}}{6 !} \phi^{6}
$$

There are three classes of diagrams in the computation of $\mathcal{M}_{2 \rightarrow 4}$, which are depicted in figure 1. In performing this computation we have to symmetrize the sums over the final momenta and take energy-momentum constraints into consideration. For $\theta=0$ these graphs add up to 0 . This is due to the fact that the shG model is integrable classically. In the deformed theory there is a non-zero contribution at order $\theta^{2}$, which means that the amplitude for particle production is non-zero. This means that the naïve Moyal deformation of the shG (and sG) model is non-integrable. This way of defining the noncommutative
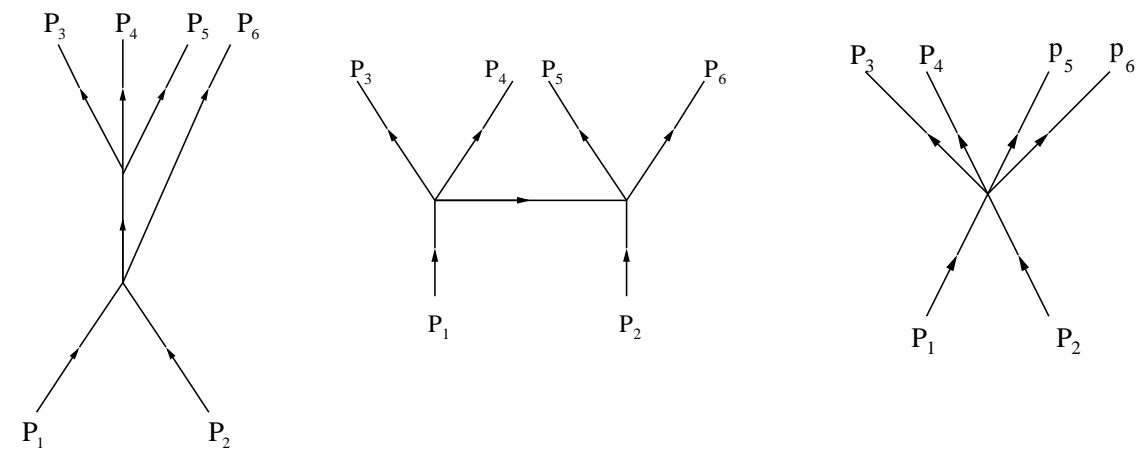

Figure 1: The three classes of diagrams contributing to $\mathcal{M}_{2 \rightarrow 4}$.

$\mathrm{sG}$ and shG models gives the following equation of motion for the noncommutative sG

$$
\partial^{\mu} \partial_{\mu} \phi+\frac{m^{2}}{\beta} \sin _{\star}(\beta \phi)=0
$$

with no further constraints.

On the other hand we can define these models through a different route such that the following two requirements are satisfied: 1 - as $\theta \rightarrow 0$ we obtain the usual sG model, and 2there is an infinite number of conserved charges by construction. We will see that this is possible to be done by generalizing the zero-curvature method to noncommutative spaces.

\section{Noncommutative Zero-Curvature Method}

If one manages to cast the equations of motion of a given two-dimensional model in the 
following form

$$
\partial_{t} U-\partial_{x} V+[U, V]=0
$$

where $U$ and $V$ are matrices depending on the fields and their derivatives, and a spectral parameter $\lambda$, and $[U, V]=U V-V U$, then it is possible to show that there is an infinite number of conserved charges in this model [i] $\left.{ }_{1}^{1} \overline{4}\right]$. When writing the equations of motion in this form, in general the diagonal terms will be the equations of motion and the offdiagonal terms vanish. We can generalize the zero-curvature condition $4.1 \mathrm{i}$ by changing the commutator of $U$ and $V$ by their $\star$-commutator

$$
\partial_{t} U-\partial_{x} V+[U, V]_{\star}=0
$$

where $[U, V]_{\star} \equiv U \star V-V \star U$. Once the equations of motion have been cast in this form, we can follow the derivation of the existence of an infinite number of conserved charges in the commuting case line by line [i] [1] $T_{\lambda}(x)$, defined as the solution of

$$
\frac{\partial T_{\lambda}}{\partial x}=U \star T_{\lambda}
$$

can be used to generate an infinite number of conserved charges. Namely, we can show from the noncommutative zero-curvature condition that $\partial_{t} \operatorname{Tr} T_{\lambda}(L)=0$, and by expanding $T_{\lambda}(L)$ in powers of the spectral parameter, we obtain the sought for conserved charges.

We will work in light-cone coordinates now, where $x_{ \pm}=\left(x_{0} \pm x_{1}\right) / 2$. It is possible to write the equation of motion for the shG model as a zero-condition equation, by introducing $A$ and $\bar{A}$,

$$
A=-\frac{m \lambda}{2}\left(e^{\beta \phi} \sigma_{-}+e^{-\beta \phi} \sigma_{+}\right) \quad \text { and } \quad \bar{A}=\frac{m}{2 \lambda}\left(\sigma_{-}+\sigma_{+}\right)-\frac{\beta}{2} \bar{\partial} \phi \sigma_{3}
$$

where $\sigma_{ \pm}=\frac{1}{2}\left(\sigma_{1} \pm i \sigma_{2}\right), \sigma_{i}$ are the usual Pauli matrices, and $\lambda$ is the spectral parameter. It is a simple computation to show that the zero-curvature condition for $A$ and $\bar{A}$

$$
\bar{\partial} A-\partial \bar{A}+[A, \bar{A}]=0,
$$

is equivalent to the equation of motion for the shG model. Notice that in showing this, the diagonal elements of the matrix equation are the equation of motion and the off-diagonal elements vanish.

We define the noncommutative shG model through the $\star$-zero-curvature equation

$$
\bar{\partial} A-\partial \bar{A}+[A, \bar{A}]_{\star}=0
$$

where $[A, \bar{A}]_{\star}=A \star \bar{A}-\bar{A} \star A$. The equation of motion derived from $\overline{4} \overline{4} \cdot \underline{\underline{\prime}}$ is

$$
\partial \bar{\partial} \phi+\frac{m^{2}}{\beta} \sinh _{\star}(\beta \phi)=0
$$

which is exactly the same equation one would obtain from the Moyal deformation of the shG action. There are, though, two more constraints, coming from the off-diagonal elements, 
and which read

$$
\begin{aligned}
& \bar{\partial}\left(e_{\star}^{-\beta \phi}\right)+\frac{\beta}{2}\left(e_{\star}^{-\beta \phi} \star \bar{\partial} \phi+\bar{\partial} \phi \star e_{\star}^{-\beta \phi}\right)=0 \\
& \bar{\partial}\left(e_{\star}^{\beta \phi}\right)-\frac{\beta}{2}\left(e_{\star}^{\beta \phi} \star \bar{\partial} \phi+\bar{\partial} \phi \star e_{\star}^{\beta \phi}\right)=0 .
\end{aligned}
$$

It is easy to show that these constraints can be written as total derivatives. Therefore the extra constraints can be seen as global conserved currents. They clearly vanish as $\theta \rightarrow 0$, when the Moyal product becomes the usual product, and we obtain the equations of motion of the shG model, as expected.

In [1] bicomplex method. In spirit this is similar to what we have done, since by construction they generate an infinite number of conserved charges, and as $\theta \rightarrow 0$ they recover the equation of motion of the sG model. They also obtained two extra constraints which can be written as total derivatives (global currents). The equation of motion found in [i] after replacing $\beta$ by $i \beta$ is

$$
\partial\left(e_{\star}^{\beta \phi} \star \bar{\partial}\left(e_{\star}^{-\beta \phi}\right)-e_{\star}^{-\beta \phi} \star \bar{\partial}\left(e_{\star}^{\beta \phi}\right)\right)=2 m^{2} \sinh _{\star}(\beta \phi)
$$

which is different from the one we found here. It is possible, though, to make a different choice of potentials for $A$ and $\bar{A}$ such that we obtain the same equation of motion. This is due to the fact that when we generalized these gauge potentials to the noncommutative case, we kept the $\partial \phi$ term intact, but this choice is not unique: we could have chosen $\frac{1}{2 \kappa}\left(e_{\star}^{-\kappa \phi} \star \partial e_{\star}^{\kappa \phi}-e_{\star}^{\kappa \phi} \star \partial e_{\star}^{-\kappa \phi}\right)$. As $\theta \rightarrow 0$ the product of fields becomes the usual product and we trivially recover $\partial \phi$. If we take $\kappa=1$, the zero-curvature condition with the gauge potentials becomes precisely the equation of motion proposed by Grisaru and Penati. It is harder to check that the same holds for the constraints. It would be very interesting to establish whether they are the same or not.

\section{Euclidean Solitons}

Now that we have the equations of motion for the noncommutative sG model we can try to solve them and see what is the generalization of the solitons in the original theory. The somewhat surprising result is that the 1-soliton solution of the sG model solves the equation of motion and constraints of the noncommutative sG model. This is due to the fact that the $\star$-product of two functions that depend linearly on the space-time variables is the same as their usual product. There are several ways to prove this, for example, consider the $\star$-product of $f\left(\sum_{\alpha} a_{\alpha} x^{\alpha}\right)$ and $g\left(\sum_{\alpha} a_{\alpha} x^{\alpha}\right)$

$$
f\left(\sum_{\alpha} a_{\alpha} x^{\alpha}\right) \star g\left(\sum_{\alpha} a_{\alpha} x^{\alpha}\right)=\left.\exp \left(\frac{i}{2} \theta^{\mu \nu} \partial_{\mu}^{z_{1}} \partial_{\nu}^{z_{2}}\right) f\left(\sum_{\alpha} a_{\alpha} z_{1}^{\alpha}\right) g\left(\sum_{\alpha} a_{\alpha} z_{2}^{\alpha}\right)\right|_{z_{1}=z_{2}=x}
$$

Expanding the exponential, using the chain rule, and the antisymmetry of $\theta^{\mu \nu}$ we see that all terms but the first vanish. This is true in any dimensions, but we are interested in the two-dimensional case only. Therefore the equation of motion of the noncommutative 
$\mathrm{sG}$ model is the same as the one of the usual sG model. Moreover, from the form of the constraints, replacing the $\star$-products by their usual product, implies the vanishing of these constraints. All this put together means that if a certain field solution depends linearly on the spacetime variables, which is the case for soliton solutions in two-dimensions, then it will also solve the noncommutative equations of motion. This establishes the fact that the 1-soliton solution of the $\mathrm{sG}$ mode is also a solution of the noncommutative sG model.

\section{Noncommutative Principal Chiral Model}

We have seen an example where the naïve Moyal deformation of an integrable field theory does not provide an integrable field theory. We will see now an example where the Moyal deformation of the action of an integrable field theory provides one, the principal chiral model $(\mathrm{pcm})$ [1] $\left.\underline{1}_{\overline{6}}^{i}\right]$.

The action of the pcm is

$$
\mathcal{S}_{p c m}=\frac{1}{2 g_{0}^{2}} \int d x \operatorname{Tr}\left(\partial^{\mu} g \partial_{\mu} g^{-1}\right)
$$

where $g$ is a groups element, belonging for example to $U(N)$ or $S U(N)$, and satisfies $g g^{\dagger}=g^{\dagger} g=1$. The Moyal deformation of the pcm is given by the action

$$
S_{p c m}^{*}=\frac{1}{2 g_{0}^{2}} \int d^{2} x \operatorname{Tr}\left(\partial^{\mu} g^{-1} \star \partial_{\mu} g\right)
$$

and the field $g$ is required to satisfy $g \star g^{\dagger}=g^{\dagger} \star g=1$. The reason why we should be specific about the group to which $g$ belongs to, is that not all groups allow noncommutative extensions, for example, there is no noncommutative $S U(N)$. Therefore we will restrict our analysis to the $U(N)$ pcm. Since the action $\overline{6} \cdot 2$ is quadratic and the manifold we are considering is trivial, the actions $\overline{3} \cdot 3 \overline{1}$ and $\overline{6} \cdot 2 \overline{1}$ are identical ${ }^{2}$. The only difference between these two models lies in the constraints. We can construct non-trivial non-local conserved charges by following the Brezin-Itzykson-Zinn-Justin-Zuber (BIZZ) method [i] $\overline{1}$ i], and we refer to [i] for this discussion.

The noncommutative zero-curvature method can be applied to the noncommutative pcm, but this time there are no extra constraints. Consider the potentials

$$
\begin{aligned}
& U(\lambda)=\frac{1}{2} \frac{l_{0}+l_{1}}{1-\lambda}-\frac{1}{2} \frac{l_{0}-l_{1}}{1+\lambda} \\
& V(\lambda)=\frac{1}{2} \frac{l_{0}+l_{1}}{1-\lambda}+\frac{1}{2} \frac{l_{0}-l_{1}}{1+\lambda},
\end{aligned}
$$

where

$$
l_{0}(x, t)=\frac{\partial g}{\partial t} * g^{-1} \quad \text { and } \quad l_{1}(x, t)=\frac{\partial g}{\partial x} * g^{-1} .
$$

Introducing this on the zero-curvature condition

$$
\frac{\partial^{2} g}{\partial t^{2}}-\frac{\partial^{2} g}{\partial x^{2}}=\frac{\partial g}{\partial t} * g^{-1} * \frac{\partial g}{\partial t}-\frac{\partial g}{\partial x} * g^{-1} * \frac{\partial g}{\partial x}
$$

\footnotetext{
${ }^{2}$ Provided the fields fall fast enough at infinity.
} 
which is the equation of motion of the pcm, and it can be rewritten in the more compact form

$$
\partial_{\mu}\left(g^{-1} * \partial^{\mu} g\right)=0 .
$$

Contrary to the noncommutative sG model, there are no further constraints in the noncommutative $U(N)$ pcm.

\section{Conclusions}

We have seen that the Moyal deformation of a given $2 \mathrm{~d}$ integrable model does not necessarily provide a integrable field theory. In the case of the sinh-Gordon model (and by replacing $\beta \rightarrow i \beta$, the sine-Gordon model) we were able to establish their non-integrability by computing the amplitude for $2 \rightarrow 4$ particles at the tree-level, and verifying it is nonzero. On the other hand, the noncommutative $U(N)$ principal chiral model defined through the Moyal deformation of the action and constraints of the $U(N)$ principal chiral model, does provide an integrable field theory, where the elegant method of Brezin et al [i1 $\left.\overline{7}_{1}\right]$ works as well as in the commutative case.

The equations of motion we found for the noncommutative sG model initially are different from the ones proposed by Grisaru and Penati in [1] $\overline{1}$ 페] . Upon a change in the definition of the noncommutative version of $\partial \phi$ we were able to find the same equation of motion as [1] 15 , but it is not trivial to establish the equality of the constraints.

We showed that the 1-soliton solution of the sine-Gordon model solves the equations of motion and constraints of the noncommutative version. It would be interesting to study the more general multi-soliton solutions.

There are several interesting directions to pursue. Initially, it would be nice to have a more thorough understanding of the conservation laws, verifying for example, that these charges are in involution. Next one could consider the noncommutative versions of different models, such as the affine Toda theories. And last but not least, the investigation of the quantization of these models is a fascinating, if somewhat difficult problem.

\section{Acknowledgments}

We would like to thank the hospitality of the Abdus Salam ICTP, where we started this work. Discussions with P. Dorey, J. Evans, A. Garcia, K. Narayan, A. Leclair, M. Neubert, V. Sahakian, M.M. Sheikh-Jabbari, and J. Varilly are gracefully acknowledged. One of us (MM) would like to thank F. Muller-Hoisen for email exchanges, and C. Wotzasek for the use of computer facilities. We also would like to thank the organizers of this workshop for the hospitality and to wish the IFT and its members many happy returns to this date. This work is in part supported by the NSF and CNPq (profix) (MM) and Fapesp (ICC).

\section{References}

[1] I. Cabrera-Carnero, M. Moriconi "Noncommutative Integrable Field Theories in 2d" [arxiv:hep-th/0211193]. 
[2] N. Seiberg and E. Witten, "String theory and noncommutative geometry", JHEP 9909, 032 (1999) [arXiv:hep-th/9908142].

[3] L. Susskind, "The quantum Hall fluid and non-commutative Chern Simons theory", [arXiv:hep-th/0101029].

[4] S. Minwalla, M. Van Raamsdonk and N. Seiberg, "Noncommutative perturbative dynamics", JHEP 0002, 020 (2000) [arXiv:hep-th/9912072].

[5] J. Gomis and T. Mehen, "Space-time noncommutative field theories and unitarity", Nucl. Phys. B 591, 265 (2000) [arXiv:hep-th/0005129].

[6] C. S. Chu, J. Lukierski and W. J. Zakrzewski, "Hermitian analyticity, IR/UV mixing and unitarity of noncommutative field theories", Nucl. Phys. B 632, 219 (2002)

[arXiv:hep-th/0201144].

[7] D. Bahns, S. Doplicher, K. Fredenhagen and G. Piacitelli, "On the unitarity problem in space/time noncommutative theories", Phys. Lett. B 533, 178 (2002) [arXiv:hep-th/0201222].

[8] T. Filk, "Divergencies in a field theory on quantum space", Phys. Lett. B 376, 53-58 (1996).

[9] M. R. Douglas and N. A. Nekrasov, "Noncommutative field theory", Rev. Mod. Phys. 73, 977 (2001) [arXiv:hep-th/0106048],

R. J. Szabo, "Quantum field theory on noncommutative Spaces", [arXiv:hep-th/0109162].

[10] A. B. Zamolodchikov and A. B. Zamolodchikov, "Factorized S-matrices in two dimensions as the exact solutions of certain relativistic quantum field models", Annals Phys. 120, 253 (1979).

[11] P. Dorey, "Exact S-matrices", [arXiv:hep-th/9810026].

[12] A. Armoni, "Comments on perturbative dynamics of non-commutative Yang-Mills theory", Nucl. Phys. B 593, 229 (2001) [arXiv:hep-th/0005208].

[13] C. Nunez, K. Olsen and R. Schiappa, "From Noncommutative bosonization to S-duality", JHEP 0007,030 (2000) [arXiv:hep-th/0005059].

[14] L. D. Faddeev and L. A. Takhtajan, "Hamiltonian methods in the theory of solitons", Berlin, Germany: Springer (1987) 592 p. (Springer Series In Soviet Mathematics).

[15] M. T. Grisaru and S. Penati, "The noncommutative sine-Gordon system", [arXiv:hep-th/0112246].

[16] S. Profumo, "Noncommutative principal chiral models", JHEP 0210 (2002) 035 [arXiv:hep-th/0111285].

[17] E. Brezin, C. Itzykson, J. Zinn-Justin, and J.-B. Zuber, "Remarks about the existence of non-local charges in two-dimensional models", Phys. Lett. B82,442-444 (1979). 